\title{
RADIO RESOURCE ALLOCATION IN MOBILE COMMUNICATION SYSTEMS
}

In the article we deal with two types of Call Admission Control Schemes (Reservation-based CAC scheme and Probability-based CAC scheme) and we made simulations in Matlab environment. Both types of these transmission schemes belong to homogeneous type of radio resource management applications. Only voice application belongs here (traditional wireless networks) and each call demands the same amount of bandwidth (channel, frequency or time slots). Both schemes are compared and evaluated at the end of the article.

\section{Introduction}

Mobile communication systems have attracted great attention during the past several years. These systems have been developed in a distributed fashion. The mobile user population has been growing at a rapid rate. More recently, the demand for multimedia application requiring high bandwidth (for example video, pictures and Web application) has increased. The core issue of providing multimedia services over a mobile wireless network is the quality of service (QoS) support in the presence of changing network connectivity due to user mobility and limited wireless communication links.

An important QoS issue in wireless multimedia networks is how to control new and handoff calls. This is a work for Call Admission Control (CAC). Another, also important issue is in Radio Resource Allocation (RRA). Both, CAC and RRA are a part of Radio Resource Management (RRM). RRM is a set of algorithms that control the usage of radio resources. It is located in user terminal, base station and radio network controller. The RRM infrastructure is described in [1]. RRM functionality is aimed to guarantee QoS, offer high capacity and maintain the planned coverage area.

\section{Radio Resource Allocation}

Radio resource allocation algorithms aim to optimal use the code-division multiple access (CDMA) capacity [2]. RRA algorithm should maximize the number of satisfied users within the available radio bandwidth. Considering the wide range of quality requirements, the RRA algorithms must perform very complex tasks.

Let $M(t)$ be the number of active mobiles in the coverage area. This number changes depending on the offered load. The number of all base stations (BSs) is $K=\{1,2 \ldots K\} . C=\{1,2 \ldots C\}$ is the number of all available channels. The gain matrix $G_{m}$, which describes the radio environment, is defined as $G_{m}=\left[G(t)_{i, j}\right]_{K x M(t)}$, where $G(t)_{i, j}$ is the link gain between the base station BSi and mobile station MSj that changes with the mobile's movement.

RRA algorithms consider the link gain matrix $G_{m}$ and perform the following tasks:

- Assign one or more (e.g., soft handoff) BS from set $B$. Call admission control algorithm decides if and where the new (or handoff) call is accepted or rejected;

- Assign one or more channels (e.g., codes for WCDMA, and combination code-timeslot for time-division CDMA, (TD-CDMA) from set $C$. The rate scheduler assigns appropriate code for the call, and the time scheduler (TS) decides when these resources can be used;

- Differentiate in resource allocation between several traffic classes. TS decides about the time moment and the amount of used resources based on the session's quality requirements.

There is a model of Radio Resource Allocation shown in Figure. 1. From the figure it is clear that a resource estimator (RE) controls the RRA algorithm represented by the dotted arrows. The solid arrows present the flow of the user information. The RE has several inputs such as the measured interference conditions, radio channel characteristics, current load in the BS, calls' traffic characteristics and QoS requirements. The RE by considering inputs performs the following control tasks:

- The radio channel characteristics and call quality requirements are used for optimal power and rate allocation;

- The current BS load, call traffic characteristics, and quality requirements are used to control the time scheduler;

- With the built-in capacity model, the RE assists CAC in accepting or rejecting new (or handoff) calls.

\subsection{RRA techniques}

There are many ways of allocating the resources, in response to a new call arrival or handoff attempt. The goal is to maximize

\footnotetext{
* Andrej Husár, Peter Čepel, Peter Brída, Vladimír Wieser

Department of Telecommunications, Faculty of Electrical Engineering, University of Žilina, Velký Diel, 01026 Žilina, Slovak Republic,

E-mail: husar@fel.utc.sk, cepel@fel.utc.sk, brida@fel.utc.sk, wieser@fel.utc.sk
} 


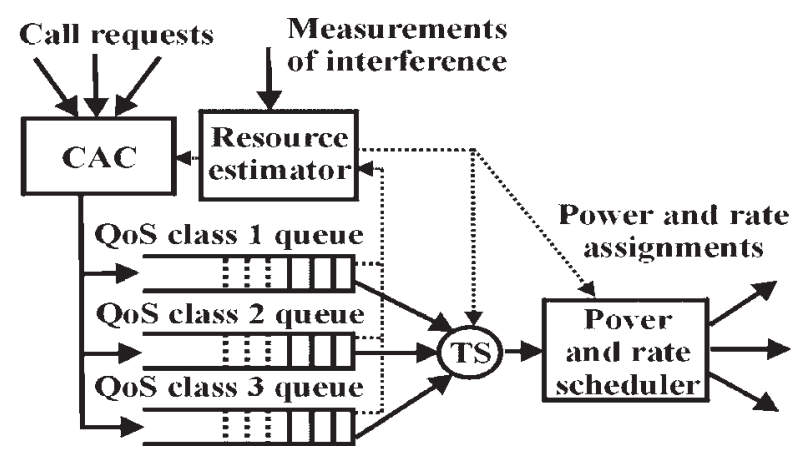

Fig. 1 RRA algorithm in the base station

system efficiency while meeting the QoS requirements of users. Some channel assignment techniques allow channel sharing among several cells. Such schemes, known as Dynamic Resource Allocation (DRA) schemes have a common resource sharing pack that can be allocated to users upon request. In DRA, channels are not permanently assigned to cells. These schemes can borrow channels from neighboring cells if necessary. However, once a channel is borrowed from a neighboring cell, all other cells are prohibited from using the channel. The other channel assignment schemes allocate a fixed amount of resources to each cell. They are referred as Static Resource Allocation (SRA) schemes.

Among RRM techniques, which are suitable for resource allocation, are the already mentioned (DRA) and SRA techniques. DRA usually co-operates with multiple access, e.g. FDMA, TDMA and with their combinations. In DRA, real time measurements of propagation and traffic conditions are used to allocate and reallocate spectrum resources. The performance of DRA schemes is very good, in particular when integrated DRA and power control is used. The performance of the DRA schemes is critically dependent on the rate at which allocation or reallocation occurs. DRA schemes have to observe the changes of signal power (at least slow fading).

A second class of allocation schemes is the class of SRA schemes. One of the representatives is Random Resource Allocation (RRA) scheme. In this scheme resource allocation is permuted in a random fashion. The simplest way to achieve this is to use orthogonal frequency hopping which can be regarded as a static allocation, where mobile terminals allocated to a certain access port swap channels with each other. Frequency hopping occurs typically 100 - 1000 times per second.

\section{Call Admission Control}

Call admission control is a method of radio resource management whose target is to adapt radio resource allocation to traffic variations. We denote $\mathrm{CAC}$ as the process to make a decision about acceptance or rejection of a new admission according to the number of available resources. We also take into consideration QoS requirements of the present users and possible effect of a new call. CAC algorithms can be divided into some groups, e.g. reservation based CAC, probability based CAC, etc. More about CAC techniques can be seen in [3].

\subsection{Reservation-based CAC scheme}

The bandwidth (or a number of channels) available in a cell must be shared by calls of the different service classes and the different service requirements, which have to be met. The partition of channels in this scheme is important and it is second in the order after CAC. A portion of channels is exclusively reserved for handover calls from neighboring cells. A next portion is reserved for real-time and non real-time data. The remaining bandwidth (channels) can be allocated on demand to voice new calls. In Fig. 2 we can see the process of resource reservation for handoff calls in the real time. Algorithm 2 computes with the greater amount of resources ( 48 channels) for handoff than algorithm 1 ( 32 channels). In our simulation we consider the QoS probability $P_{q o S}=0.001$. Fig. 3 shows the total number of free channels for new calls and data transfer, which depends on the reserved resources for handoff.

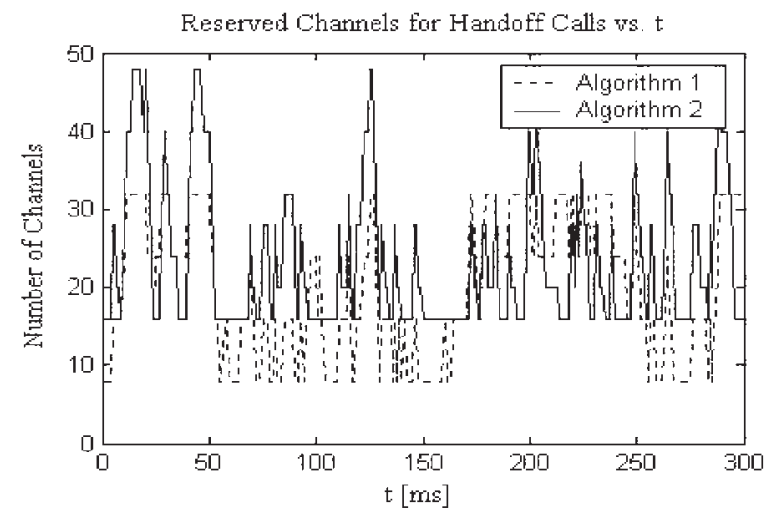

Fig. 2 Reserved channels for handoff calls

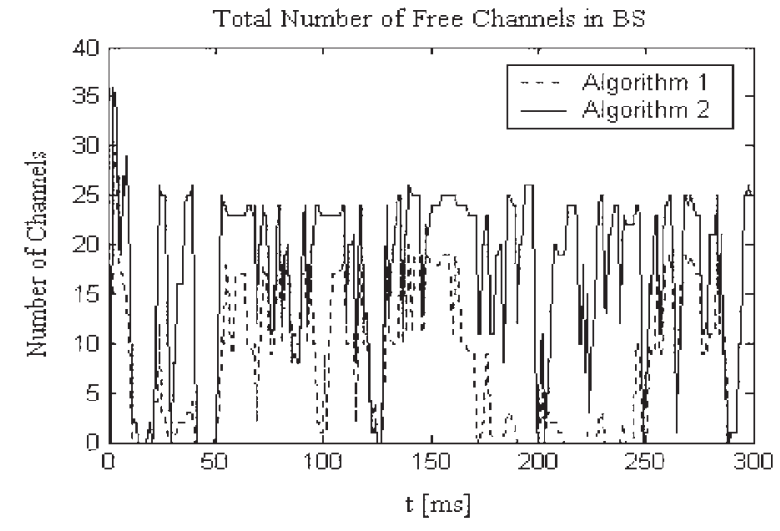

Fig. 3 Total number of free channels in BS 


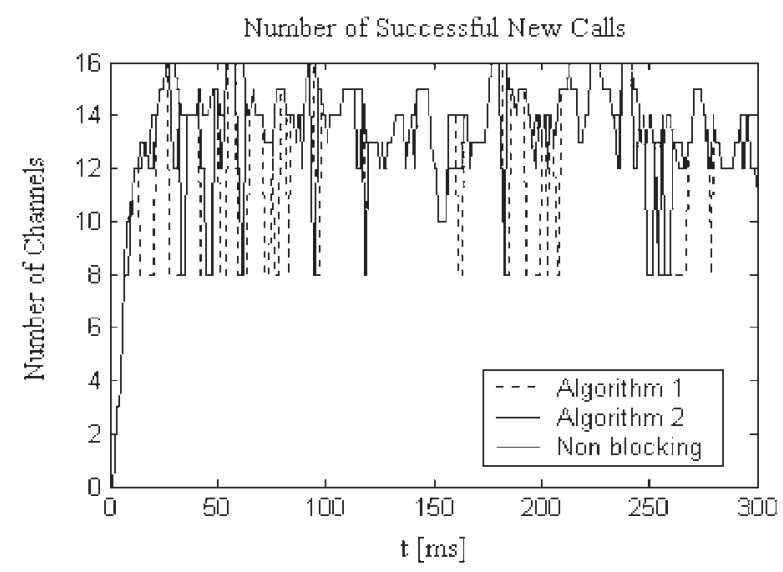

Fig. 4 Number of successful new calls

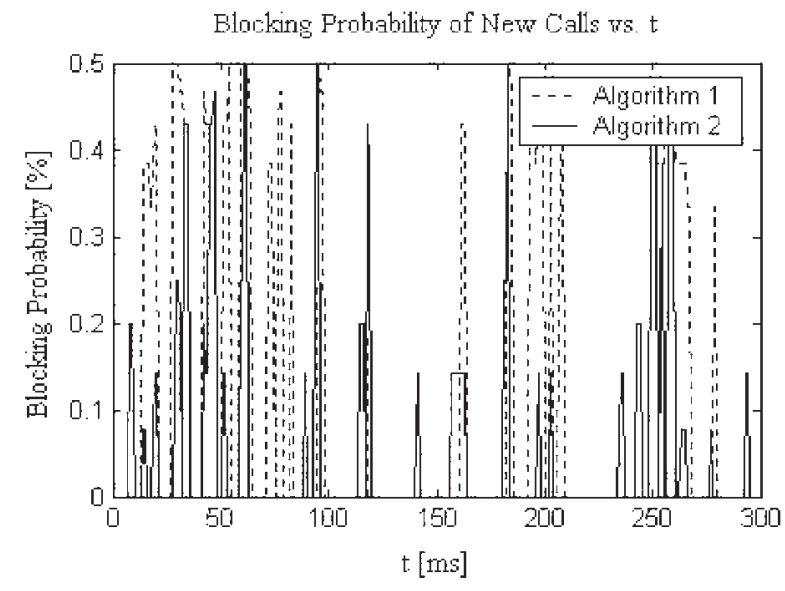

Fig. 5 Blocking probability of new calls

\subsection{Probability-based CAC scheme}

In this scheme, the amount of reserved bandwidth (channels) for handoff calls and new calls is determined according to a call dropping probability measured during the last few time [4]. However, if this measurement time range is short, accuracy of the call dropping probability is not good. In this scheme we can estimate a call dropping probability. It is possible to manage free resources in the main cell and in the neighboring cells according to a given dropping probability. In Fig. 4 is the time behavior of the number of successful new calls in the main cell. It depends on the probability of dropped calls and on the reserved resources for handoff calls. When the system has limited resources due to handoff process, blocking probability of new calls increases (Fig. 5). The amount of the blocking probability depends on the used algorithm and free resources in the current cell.

\section{Conclusion}

In this paper, we describe the basis of radio resource allocation and two techniques of CAC. This is an important part of

adaptive radio resource management in mobile communication systems. We have simulated two CAC techniques in the Matlab environment to illustrate the radio resource utilization in a sevencell model of the mobile system.

In the reservation-based CAC scheme free resources for new calls and data applications depend on the handoff calls and on the amount of resources reserved for these calls. In the probabilitybased CAC scheme free resources depend on dropping probability of the handoff calls. These calls are dropped in the handover process among the main cell and neighboring cells.

\section{Acknowledgements:}

The authors gratefully acknowledge support from the VEGA project No. 1/0140/03 "Effective radio resources management methods in next generations of mobile communication networks" and State project No. 2003 SP 51/028 09 00/028 0910 "Communication Networks and Services of New Generations”.

\section{References}

[1] HOLMA, H., TOSKALA, A.: WCDMA for UMTS: Radio Access for Third Generation Mobile Communication, John Wiley, 2001, http://citeseer.nj.nec.com.

[2] JORGUSESKI, L., FLEDDERUS, E., FARSEROTU, J.: Radio Resource Allocation in Third-Generation Mobile Communication Systems, IEEE Communications Magazine, February 2001, pp. 117-123.

[3] HUSAR, A., WIESER, V.: Call Admission Control Schemes for Cellular Networks, Radioelektronika - International Czech \& Slovak Scientific Conference, 2003, pp. 64-67.

[4] MA, Y., HAN, J. J., TRIVEDI, K. S.: Call Admission Control for Reducing Dropped Calls in Code Division Multiple Access Cellular Systems, IEEE INFOCOM 2000, pp. 1481-1490, 2000. 